\section{Recomendaciones para el uso correcto de densitometría ósea en la práctica clínica. Consenso de la Sociedad Chilena de Endocrinología y Diabetes}

\author{
MARCELA BARBERÁN M. ${ }^{1,2}$, CLAUDIA CAMPUSANO M. ${ }^{3,4}$, \\ PATRICIO TRINCADO M. ${ }^{2}$, SOFÍA OVIEDO G. ${ }^{5}$, \\ SERGIO BRANTES G. ${ }^{6},{ }^{2}$ JORGE SAPUNAR Z. ${ }^{7}$ \\ PARTICIPANTES: JOSÉ CANESSA ${ }^{8}$, PÍA CID ${ }^{4}$, FREDDY ESCOBAR ${ }^{2}$, \\ DANIELA EUGENIN ${ }^{1,3,4}$, PABLO FLORENZANO ${ }^{9}$, HÉCTOR GAJARDO ${ }^{10}$, \\ GILBERTO GONZÁLEZ ${ }^{1}$, FRANCISCA ILLANES ${ }^{11}$, BEATRIZ JIMÉNEZ ${ }^{12}$, \\ CAROLINA MARTÍNEZ ${ }^{2}$, EDITH MIRANDA ${ }^{2}$, SANDRA RIVERA ${ }^{5}$, \\ PATRICIO SALMAN ${ }^{13}$, PAMELA TREJO ${ }^{11}$, SOLEDAD VELASCO ${ }^{11}$
}

\section{Guidelines of the Chilean Endocrinology Society for the correct clinical use of bone densitometry}

Osteoporosis is a silent and frequent disease, which increases fracture risk. Approximately half of women and one of five men over 50 years old will suffer an osteoporotic fracture throughout their lives. Dual-energy $x$-ray absorptiometry (DXA) allows a real bone mineral density (BMD) measurement in different parts of the skeleton and is considered the "gold standard" for quantifying osteoporosis with high accuracy and precision. The Board of the Chilean Society of Endocrinology and Diabetes (SOCHED) required from the Bone Disease Study Group to develop a consensus about the "Correct use of bone densitometry in clinical practice in Chilean population". Therefore, we elaborated 25 questions which addressed key aspects about the indications for a DXA scan, and the details of how to perform and report this test. Since some of the evidence obtained was of low quality or inconclusive, we decided to create a multidisciplinary group of national experts in osteoporosis to develop a consensus in this subject. The group consisted of 22 physicians including endocrinologists, gynecologists, geriatricians, radiologists, rheumatologists and nuclear medicine specialists. Using the Delphi methodology to analyze previously agreed questions, we elaborated statements that were evaluated by the experts who expressed their degree of agreement. The final report of this consensus was approved by the SOCHED board.

(Rev Med Chile 2018; 146: 1471-1480)

Key words: Absorptiometry, Photon; Consensus; Densitometry; Osteoporosis, Postmenopausal; Practice Guideline.

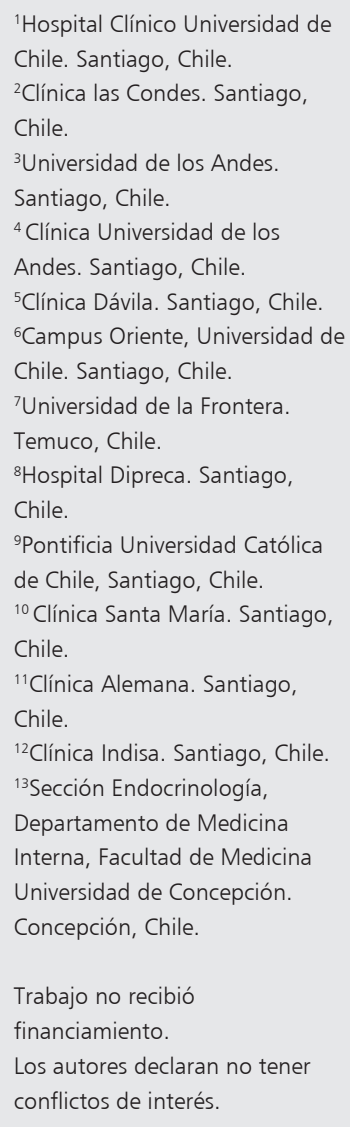

L a osteoporosis es una patología frecuente, a menudo silente, e implica un riesgo aumentado de fracturas con mínimo trauma. Esta patología constituye un problema de salud pública, dado su prevalencia y los costos asociados. A nivel global se ha estimado que aproximadamente la mitad de las mujeres mayores de 50 años y 1 de cada 5 hombres, sufrirán una fractura osteoporótica a lo largo de su vida ${ }^{1}$.

La densitometría ósea (DO) permite la me- 
dición de la densidad mineral ósea (DMO) en diferentes partes del esqueleto, con el objetivo de realizar el diagnóstico de osteoporosis, predecir el riesgo de fractura, tomar decisiones terapéuticas y evaluar la respuesta a tratamiento. Es considerada el gold standard para diagnosticar osteoporosis con muy buena exactitud (ROC $0,65-0,9$ ) y precisión (1-2\%), siendo aprobado para su uso en el año 1988 por organismos internacionales ${ }^{2,3}$.

Existen diferentes métodos de evaluación de la DMO, como la absorciometría de doble energía de rayos X (DXA), el más usado, tomografía computada de alta resolución (QCT), ultrasonido cuantitativo (QUS), entre otros. El principio básico de la tecnología DXA es la medición de tejidos vivos a través de un haz de rayos $\mathrm{X}$ de dos niveles de energía diferentes, lo que dependerá de los tejidos atravesados y de la energía del haz de radiación, siendo detectados por un sensor ubicado en el extremo opuesto del paciente. Los sistemas DXA consideran dos tipos de tejidos: hueso y tejidos blandos. La intensidad residual del haz de rayos X medida en el detector, se compara con la densidad conocida de los valores de referencia del equipo. Los resultados se expresan en masa por unidad de área $\left(\mathrm{g} / \mathrm{cm}^{2}\right)$, que se denomina densidad areal (Figura 1) $)^{4,5}$.

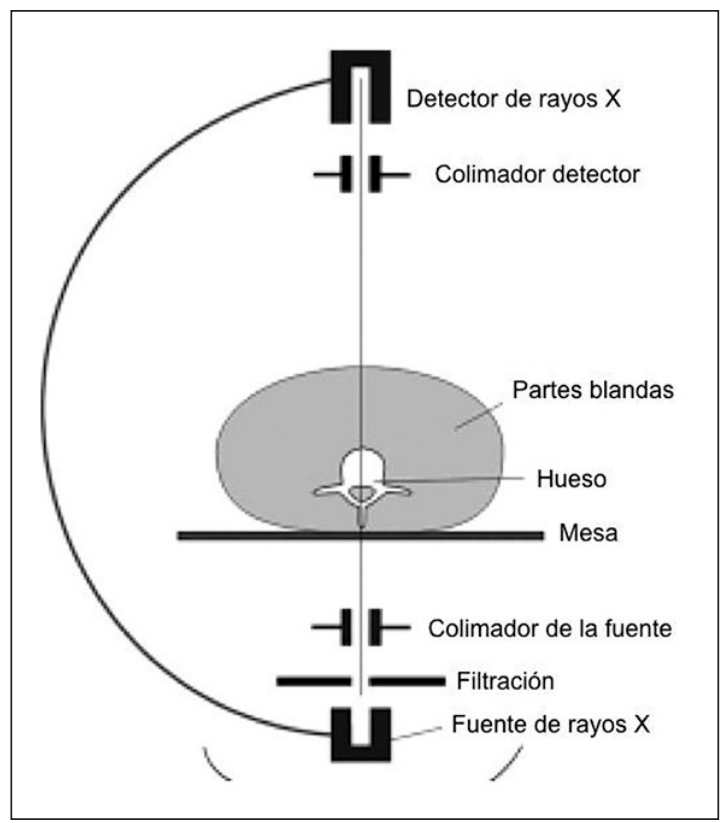

Figura 1. DXA (Absorciometría de Doble energía de rayos $X$ ).
En el procesamiento del examen con el equipo DXA se reconstruyen las imágenes radiológicas de los sitios explorados. En estas se delimitan las áreas de interés (ROI, region of interest), y se calcula la densidad areal. Este valor se compara contra una base de datos normalizada, que actualmente es NHANES III (National Health and Nutrition Examination Survey III), comparando al paciente con sujetos del mismo sexo y etnia, separadamente por la misma edad (Z-score) y por la edad en que se obtiene la masa ósea máxima (T-score $)^{6}$.

La DO tiende a ser erróneamente considerada como una técnica rutinaria y automatizada. Sin embargo, requiere una indicación apropiada, metodología cuidadosa e interpretación precisa, lo cual sólo es posible con la adecuada formación e interacción entre los encargados de la realización e informe del examen y médicos clínicos que los solicitan ${ }^{4}$. La Sociedad Chilena de Endocrinología y Diabetes (SOCHED) ha convocado a expertos nacionales con el fin de redactar un documento de consenso para el uso correcto de densitometría ósea en el diagnóstico de pacientes adultos adaptado a la realidad nacional y que esperamos sea de ayuda para los médicos y tecnólogos médicos que se enfrentan con esta herramienta diagnóstica.

\section{Metodología}

El Directorio de la SOCHED solicitó al Grupo de Estudio de Enfermedades Óseas el desarrollo de un pronunciamiento respecto al "Uso correcto de densitometría ósea en la práctica clínica" en Chile. Para ello, se constituyó un comité de coordinación integrado por 2 miembros del Grupo de Estudio que elaboró 25 preguntas que abordaban aspectos claves de la indicación del examen, así como de su realización y del informe. Una vez aprobadas por el Grupo de Estudios de Enfermedades Óseas, se intentó contestar las preguntas mediante una revisión sistemática (MEDLINE, Cochrane). Dado que parte de la evidencia obtenida resultó de baja calidad o no concluyente se decidió incluir en el pronunciamiento un consenso de expertos. Para ello, el comité de coordinación seleccionó a 22 expertos o especialistas nacionales (endocrinólogos, ginecólogos, geriatras, radiólogos, reumatólogos, especialistas en medicina nuclear) con experiencia en el área de la osteoporosis.

A partir de las preguntas previamente acorda- 
das se elaboraron afirmaciones para metodología Delphi acompañadas de una escala visual de Likert con 5 opciones en la que cada experto expresara su grado de acuerdo.

Definimos consenso cuando más de $66 \%$ de los expertos estuvieron de acuerdo o en desacuerdo con la afirmación. Si no se lograra consenso en primera ronda, el proceso se repite informándoles el resultado y continuando hasta lograr acuerdo/ desacuerdo o que la proporción de respuestas no experimente variación. El nivel de consenso se clasificó en grado U (100\%), grado A (90-99\%), grado B (78-89\%), y grado C (67-77\%). Con nivel de consenso U-A, el panel de expertos recomendó ejecutar o no ejecutar la acción implícita en la afirmación. Con nivel de consenso B-C el panel sólo sugirió.

Para aquellas preguntas cuyas respuestas se fundamentan en evidencia de calidad regular, se utilizaron los términos "sugerimos" o "no sugerimos" y el número 2 según metodología GRADE. Cuando la evidencia fue de buena calidad se utilizaron los términos "recomendamos" o "no recomendamos" y el número 1 . El informe final de este consenso fue aprobado por Directorio de la SOCHED .

\section{Resultados}

\section{Tamizaje y diagnóstico}

\section{1. ¿Cuáles son las indicaciones de densitometría ósea?}

Se recomienda tamizaje con densitometría ósea en mujeres posmenopáusicas mayores de 65 años, independiente de los factores de riesgo (Recomendación, GRADE 1).

Esta recomendación ha sido propuesta por su costo-efectividad y beneficio en este grupo etario. Recientemente en el estudio SCOOP (Screening for prevention of fractures in older women), se evaluó el efecto del tamizaje de osteoporosis en la incidencia de fracturas a 5 años, usando FRAX y la probabilidad de fractura de cadera a 10 años, demostrando una reducción en riesgo de fractura de cadera (HR 0,72; IC 95\% 0,59-0,89), no así en otros tipo de fracturas, con mínimos efectos en calidad de vida ${ }^{7}$.

Se sugiere tamizaje en mujeres posmenopáusicas entre 50 y 64 años y uno o más factores de riesgo de osteoporosis: IMC menor a $20 \mathrm{~kg} / \mathrm{m}^{2}$, padres con fractura de cadera, tabaquismo activo, alcohol $3 \mathrm{o}$ más dosis por día, y/o FRAX (Chile) mayor o igual a 3,2\% en riesgo de fracturas osteoporóticas mayores a 10 años (Consenso, Grado C).

Una revisión sistemática estudió diferentes herramientas de riesgo validadas. Todas tuvieron un rendimiento moderado, sin diferencias entre ellas9. USPSTF (United States Preventive Services Task Forces) propuso el uso del FRAX, que establece que una mujer mayor de 65 años sin factores de riesgo tiene $9,3 \%$ de probabilidad de presentar una fractura osteoporótica a 10 años. Por lo tanto, toda mujer de 50-64 años con un riesgo mayor o equivalente a ese, se beneficia de una DO. Estos datos se extrapolan para el cálculo del porcentaje umbral sugerido para realizar tamizaje en este consenso nacional. Es decir, toda mujer de 50-64 años con un riesgo mayor o equivalente a 3,2\% en nuestro país se beneficiaría de tamizaje con DO.

Se sugiere tamizaje en hombres mayores de 70 años con uno o más factores de riesgo de osteoporosis: IMC menor a $20 \mathrm{~kg} / \mathrm{m}^{2}$, padres con fractura de cadera, tabaquismo actual, alcohol 3 o más dosis por día (Consenso, Grado B).

Algunas guías sugieren tamizaje en todos los hombres mayores de 70 años, y entre 50 y 70 años con uno o más factores de riesgo ${ }^{1}$, sin embargo, este punto es controvertido?.

No se recomienda tamizaje en mujeres premenopáusicas, posmenopáusicas entre 50 -64 años ni en hombres sin factores de riesgo ${ }^{1}$ (Recomendación, GRADE 1).

\section{Indicaciones de densitometría ósea en otras situaciones clínicas}

Se recomienda en mujeres y hombres mayores de 50 años en que se documente fractura por fragilidad para establecer la gravedad de osteoporosis o pérdida ósea (Consenso, Grado A).

Según guías AACE (American Association of Clinical Endocrinologists) se define como fracturas por fragilidad ósea aquellas que ocurren en columna o cadera ante trauma mínimo (por ejemplo una caída desde la posición de pie a nivel del suelo) independiente de la densidad mineral ósea. También consideran las fracturas de húmero proximal, pelvis o antebrazo distal asociadas a "osteopenia", aunque otras guías no las incluyen. Se excluyen de esta definición las fracturas asociadas a trauma mayor en cualquier otra localización, en 
especial las de los dedos y craneofacial ${ }^{1}$. En cuanto a otros sitios (costillas, tibia/peroné, clavícula), no hay una recomendación para su definición como osteoporosis clínica ${ }^{10}$.

Otros grupos de expertos consideran las fracturas de columna clínicas o radiológicas como fracturas por fragilidad ósea cuando están asociadas a "osteopenia densitométrica" y a un trauma de baja energía ${ }^{10}$.

Se recomienda en mujeres y hombres como estudio basal y en seguimiento de condiciones médicas $o$ fármacos asociados a osteoporosis secundaria $o$ pérdida ósea.

Se recomienda en diabetes mellitus 1 y 2 , artritis reumatoide, síndromes de malabsorción o malnutrición, hipertiroidismo, hiperparatiroidismo primario, hipogonadismo o insuficiencia ovárica prematura, amenorrea de más de 6 meses de evolución ${ }^{11}$, osteogénesis imperfecta del adulto, hepatopatía crónica, insuficiencia renal crónica (Recomendación, GRADE 1), espondiloartropatías en mayores 50 años ${ }^{12}$ (Consenso, Grado A), cirugía bariátrica (basal y/o 2 años posterior) ${ }^{13}$ (Consenso, Grado A).

Se recomienda en usuarios de glucocorticoides (dosis equivalente a $5 \mathrm{mg}$ de prednisona al día o más por un período mayor a 3 meses), inhibidores de aromatasa, agonistas y antagonistas de $\mathrm{GnRH}$, tiazolidinedionas con 1 o más factores de riesgo de osteoporosis (Recomendación, GRADE 1), inhibidores de calcineurina como ciclosporina y tacrolimus (previo a trasplante renal ) ${ }^{16}$ (Consenso, Grado A).

Se sugiere en enfermedad bronquial obstructiva crónica en mayores de 50 años ${ }^{14}$ (Consenso, Grado B), infección por VIH (en mujeres posmenopáusicas y hombres mayores de 50 años, entre 40 y 49 años con 1 o más factores de riesgo $)^{15}$, (Consenso, Grado C).

Se sugiere en usuarios de antiepilépticos de uso crónico (Consenso, Grado C) ${ }^{16}$, inhibidores de bomba protones en tratamiento crónico (Consenso, Grado B ), inhibidores selectivos de la recaptación de serotonina (tratamiento crónico con $1 \mathrm{o}$ más factores de riesgo de osteoporosis) (Consenso, Grado B), heparina en tratamiento crónico (Consenso, Grado $C$ ). Se ha comunicado mayor riesgo en uso por períodos mayores a 6 meses.

Se recomienda en mujeres y hombres en quienes se documente "osteopenia" en radiología convencional (Recomendación, GRADE 1)1.
Se recomienda para valorar la efectividad de terapia farmacológica antiosteoporótica (Recomendación, GRADE 1) ${ }^{1}$.

Se sugiere no solicitar densitometría ósea en pacientes con déficit de vitamina $D$ aislado, es decir, no asociado a hiperparatiroidismo secundario (Consenso, Grado C).

2. ¿Cuáles son los factores de riesgo de osteoporosis y cuándo usarlos?

El FRAX es una herramienta que integra una serie de factores de riesgo clínicos, pudiendo o no incorporar DMO de cuello femoral, para calcular la probabilidad de fractura osteoporótica mayor (columna, antebrazo, húmero o cadera) o de cadera a 10 años $^{1,11}$. Su uso sin DO permite seleccionar a pacientes a quienes solicitar el examen (umbral diagnóstico) y con DO determina los pacientes a tratar según estimación de riesgo de fractura (umbral de tratamiento). Lo ideal es utilizar umbrales basados en población local ${ }^{8}$.

Se recomienda utilizar el score FRAX como herramienta de tamizaje en mujeres posmenopáusicas y hombres mayores de 50 años (Consenso, Grado C).

\section{Definición osteoporosis densitométrica y criterios diagnósticos}

Se recomienda definir osteoporosis densitométrica, cuando hay un T-score igual o menor a $-2,5$ en columna lumbar, cuello femoral, cadera total $y / 0$ radio 33\%, es decir, densidad mineral ósea igual o bajo 2,5 desviaciones estándar del valor para una población joven. La medición del radio 33\% se reserva solo para cuándo la DMO no puede ser medida o no es interpretable en columna o cadera, y en casos de hiperparatiroidismo primario y secundario (Recomendación, GRADE 1) $)^{1,6,17}$.

Se sugiere definir osteoporosis clinica ante la presencia de fracturas por fragilidad, en ausencia de otra enfermedad metabólica ósea, en columna, cadera o cuello femoral, en una mujer posmenopáusica u hombre mayor de 50 años independiente del resultado de la densitometría ósea. (Consenso, grado C).

No hubo consenso a favor ni en contra, en definir osteoporosis en presencia de fracturas por fragilidad en húmero proximal, pelvis y antebrazo distal asociado a osteopenia ${ }^{1,10}$.

\section{4. ¿Cómo calcular el T y Z-score?}

Se sugiere para calcular el T-score, tanto de mujeres como de hombres, utilizar como referencia 
una población femenina entre 20-29 años, caucásica (NHANES III) (Consenso, grado B) ${ }^{18}$.

Se recomienda fuertemente para calcular el $Z$-score utilizar como referencia la población específica ajustada por edad, sexo y etnia (Consenso, grado $U)^{18}$.

Se recomienda utilizar el $T$-score para informar la densidad mineral ósea de mujeres posmenopáusicas y hombres mayores de 50 años; se recomienda utilizar el Z-score para informar la densidad mineral ósea de niños, mujeres premenopáusicas $y$ hombres menores de 50 años (Recomendación, GRADE 1$)^{6}$.

Un valor $Z$ inferior a -2,0 indica una "densidad mineral ósea baja para la edad", sin embargo, el criterio densitométrico aislado no debe ser usado para diagnóstico de osteoporosis en estas poblaciones y debe asociarse a evaluación clínica.

No se recomienda utilizar el T-score para informar la densidad mineral ósea de niños, mujeres premenopáusicas y hombres menores de 50 años (Recomendación, GRADE 1)6.

5. ¿Qué otras utilidades tiene la densitometría ósea además del diagnóstico de osteoporosis?

a. Composición corporal total. En la actualidad es considerado el gold estándar para la identificación de sarcopenia en clínica ${ }^{19}$.

b. Score de hueso trabecular, (TBS, Trabecular Bone Score): es una medición de la graduación de los grises de un análisis de DXA de columna anteroposterior, mediante un software específico (TBS iNsight ${ }^{\circledR}$ ) que se utiliza como complemento de DXA y proporciona un índice de la microarquitectura ósea. Se ha demostrado que los valores bajos tienen capacidad para predecir fracturas en pacientes con valores osteopénicos o normales de DMO. Es especialmente útil en la evaluación de pacientes con osteoporosis secundaria ${ }^{21}$. Se utiliza también para mejorar la predicción del FRAX.

c. Evaluación de fracturas de columna, (VFA, Vertebral fracture assesment): es una excelente metodología para identificar fracturas vertebrales moderadas o graves, con exactitud diagnóstica e indicaciones similares a la radiografía de columna dorso lumbar lateral. Sus ventajas son la exposición a menor radiación ionizante y la realización simultánea a la DXA. Se ha recomendado usar el análisis semi-cuantitativo de Genant para el diagnóstico de frac- turas vertebrales con VFA y para confirmar la severidad, utilizar el análisis morfométrico adicional $^{22}$.

\section{Técnica densitométrica}

1. ¿Qué métodos de evaluación ósea existen?

Los equipos DXA se pueden dividir en axial y periférico según la zona de exploración. Los DXA axial tienen capacidad segmentaria para evaluar caderas, columna lumbar, antebrazo y esqueleto total. Los equipos periféricos sólo evalúan muñeca o calcáneo ${ }^{4}$.

Los equipos DXA axial cuentan con control de calidad estandarizado que permite comparar con seguridad la evolución de la masa ósea. Los de última generación (ej. GE Lunar iDXA ${ }^{\oplus}$ y Hologic Horizon $^{\varpi}$ ) tienen una elevada exactitud y precisión, imágenes de mejor resolución, menor radiación y menor tiempo de adquisición del examen.

Los equipos periféricos presentan mayor variabilidad en los resultados, poca estandarización y ausencia de datos de referencia validados. Tienen como principal ventaja su menor costo, mayor portabilidad y en el caso de los equipos basados en ultrasonido, la ausencia de radiación ionizante. En la actualidad, se aceptan como método de tamizaje, pero no permiten establecer por sí mismos diagnóstico, seguimiento ni monitorizar terapia ${ }^{4}$. Entre otras técnicas cabe destacar el QUS que evalúa el esqueleto periférico, se utiliza como método de tamizaje, pero tiene escasa correlación con DXA.

Por otra parte, el QCT y la resonancia magnética de alta resolución permiten la determinación cuantitativa de la masa ósea del hueso trabecular, cortical axial y periférico. Sus limitaciones son la alta radiación ionizantes en QCT y su alto costo ${ }^{5}$. Por último, entre las técnicas invasivas están la biopsia ósea simple con o sin histomorfometría ósea.

\section{2. ¿Cuáles serían las contraindicaciones y medi- das de seguridad?}

No hay contraindicaciones absolutas para realizar DXA. Existen contraindicaciones relativas: embarazo $^{6} \mathrm{o}$ incapacidad del paciente de mantener una posición correcta para la adquisición de la imagen. Otra limitación es cuando el peso del paciente excede el límite superior del equipo. 
Se sugiere en el caso de haber utilizado medio de contraste yodado endovenoso, reprogramar después de 1 semana de la realización del examen (Consenso, grado C). En caso de bario gastrointestinal, reprogramar para 2 semanas después de la administración del contraste (Consenso, grado B).

No hubo consenso a favor ni en contra en posponer el examen posterior a uso de gadolinio u otros radioisótopos.

Se sugiere no ingerir tabletas de calcio en las $24 \mathrm{~h}$ previas al examen (Consenso, grado B).

Existen limitaciones técnicas como el uso previo de medios de contraste. Entre estos deben considerarse bario, contraste yodado endovenoso, gadolinio y otros radioisótopos. Hay comunicaciones de aumento de la DMO posterior a administración de bario y medios de contraste yodados hasta 7 días después de su administración, sin demostrar cambios con el uso de gadolinio. El efecto de los otros radioisótopos depende del tipo, dosis, localización anatómica y de la marca del equipo DXA ${ }^{4,20}$.

3. ¿Debe tener alguna certificación el tecnólogo que realiza y el médico que informa la densitometría?

Dado que en Chile no existen normas relacionadas con la certificación, se recomienda fuertemente que el examen sea realizado por un tecnólogo médico con conocimientos y habilidades específicas en densitometría ósea (Consenso, grado U).

Además, se recomienda fuertemente que debe ser interpretado y realizado bajo la supervisión de un médico con conocimientos específicos en densitometría ósea (Consenso, grado U).

\section{4. ¿Cómo calibrar el equipo?}

La calibración es parte fundamental del control de calidad del equipo. Consiste en medir un estándar de referencia o fantoma con densidad conocida que simula los puntos anatómicos medidos en clínica. Se compara el valor medido con el valor registrado del fantoma y, de esta forma, calcula la desviación de la medición. El objetivo de esta calibración, es asegurar la "exactitud" del equipo.

Se recomienda fuertemente que cada centro realice diariamente la calibración del equipo DXA antes de la medición del primer paciente del día (Consenso grado $U)^{5,7}$. Se sugiere la calibración al menos 1 vez por semana para los equipos de última generación (como por ej. iDXA ${ }^{\oplus}$ Hologic Horizon ${ }^{\oplus}$ ) (Consenso, grado C) $)^{6}$.
5. ¿Qué significa “precisión” y "exactitud” del densitómetro?

Se sugiere determinar el "error de precisión" y calcular el "cambio mínimo significativo" (LSC) para cada equipo DXA. No se sugiere usar el error de precisión otorgado por el fabricante del equipo (Consenso, grado C).

Se sugiere expresar la "precisión" en términos de porcentaje de coeficientes de variación (\%CV) (Consenso, grado B).

La "precisión" mide la capacidad del sistema de reproducir un mismo resultado cuando el procedimiento es repetido de un modo similar en el tiempo y sin cambios biológicos reales. Esto es fundamental para determinar el mínimo cambio significativo (LSC en inglés) que significa un cambio clínicamente relevante.

La precisión debe ser determinada por cada tecnólogo por mediciones repetidas en una serie de pacientes ( 15 pacientes 3 veces o 30 pacientes 2 veces). Su valor oscila entre 2 y $5 \%$ para la mayoría de los equipos y operadores con experiencia. La precisión de un sistema debe ser establecida para cada equipo, su operador y sitio anatómico explorado 6 .

La "exactitud" refleja que tan aproximado al valor real es la medición efectuada. Se expresa como la diferencia entre el valor real y el valor medido, en términos porcentuales. En general, un sistema DXA tiene un error de exactitud menor a $10 \%$.

\section{Interpretación de densitometría}

1. ¿Qué sitios son recomendados para medición de densidad ósea? ¿Cuándo indicar cada sitio?

Se recomienda medir la densidad ósea en la columna lumbar (los cuerpos vertebrales de L1 a L4) en proyección anteroposterior y ambas caderas (cuello femoral $y / 0$ cadera total) en todos los pacientes para diagnóstico de osteoporosis (Recomendación, GRADE 1) $)^{1,6}$.

Deben ser excluidas las vértebras con secuelas de fractura o lesión focal y/o las que tengan más de 1 desviación estándar de diferencia con el resto del segmento. Si no se pueden analizar al menos dos vértebras contiguas, el estudio lumbar no es valorable. En el estudio de caderas se puede medir cuello femoral, cadera total, Ward, trocánter. Se recomienda usar sólo cuello de fémur y/o cadera 
total e idealmente de ambos lados. Serán descartadas las caderas con secuelas de fractura, lesión focal o prótesis. El valor determinante es el más bajo de los dos sitios.

No se recomienda usar el valor de "cadera bilateral promedio" para diagnóstico. Se sugiere usar este sitio para monitoreo de tratamiento, aunque en casos de asimetría de densidad entre ambas caderas se sugiere realizar seguimiento individualizado (Recomendación, GRADE 1)6.

No se recomienda usar otras regiones de interés de la cadera incluyendo el área de Ward o el trocánter para diagnóstico ni para seguimiento, por su gran variabilidad (Recomendación, GRADE 1) ${ }^{1,6}$.

Se recomienda la medición en antebrazo. En este sitio se puede medir radio ultradistal, distal, 33\% o 1/3 y cúbito. Para efectos de diagnóstico sólo se utiliza radio $33 \%$ en antebrazo no dominante. Esta medición es útil en situaciones específicas como hiperparatiroidismo, situaciones que dificultan una adecuada obtención de valores de densidad en columna y/o caderas (por ejemplo prótesis, osteosíntesis, artrosis, obesidad mórbida, etc) (Recomendación, GRADE 1) .

2. ¿Qué es una adecuada calidad de medición desde el punto de vista del médico que lo interpreta?

La calidad de la medición dependerá de factores dependientes del equipo (ver Técnica densitométrica), del paciente y de la imagen obtenida.

Se recomienda una adecuada posición en la camilla del paciente, para obtener imágenes confiables (Recomendación, GRADE 1)5.

En el estudio de columna lumbar anteroposterior, el paciente se sitúa en decúbito supino con las rodillas flexionadas sobre un soporte que reduce la lordosis y acerca la columna a la mesa de exploración y debe estar recta y centrada. El campo de visión debe incluir 1-2 cm por encima y por debajo del área que se va a analizar (entre vértebra T12 y L5, visualizando el margen superior de las crestas ilíacas). En el estudio de cadera, el paciente se coloca en decúbito supino con la pierna ligeramente en abducción para mantener recto el eje femoral, y en rotación interna (15-30º), de manera que, en la imagen adquirida, el trocánter menor no sea visible. Al situar la región de interés para medir cuello femoral, esta no debe abarcar la superposición de la rama isquiopubiana ni el trocánter mayor. Para antebrazo, el paciente se sienta al lado de la mesa con el antebrazo apoyado en ella, con la mano en pronación y sujeta con una banda. En este sitio, el área de análisis se sitúa en la región distal del radio, específicamente radio $33 \%{ }^{4}$.

El software permite adquisición y delimitación automática de las áreas de interés y rebordes óseos, pero también permite el procesamiento manual de las imágenes que puede corregir algunos errores sobretodo de las de limitaciones. La información básica aportada al equipo como edad, peso, talla, género, etnia, deben ser correctas ya que pueden inducir a error en los resultados obtenidos. El posicionamiento correcto en la camilla que se obtiene con ayuda del sistema óptico del equipo. El operador deberá verificar que se cumplan los estándares de aceptación del equipo ${ }^{3,4}$.

3. ¿Cuáles son los artefactos o factores que alteran la interpretación de la densidad ósea en columna, caderas o antebrazo?

Se recomienda evaluar los errores relacionados al equipo y al operador (Recomendación, GRADE 1) $)^{5,6}$.

Se recomienda fuertemente aplicar un cuestionario para detectar los artefactos dependientes del paciente que pueden influenciar los resultados de la densitometría ósea (Consenso, grado U).

Los artefactos posibles que pueden falsear la medición $\operatorname{son}^{4-6}$ :

a. hiperdensos: elevan falsamente la densidad ósea como por ejemplo cambios degenerativos osteoarticulares en columna y caderas, aplastamientos vertebrales, escoliosis, calcificaciones vasculares o metastásicas, cuerpos extraños (prótesis metálicas, silicona, material de osteosíntesis, stents vasculares, balas, calcificaciones heterotópicas, piercing, joyas, billeteras, etc.) Otros elementos son la presencia de tabletas de calcio y los medios de contraste radiológicos.

b. hipodensos: disminuyen falsamente la densidad ósea como laminectomía, metástasis líticas, quistes óseos, etc.

c. artefactos por excesivo tejido blando: una excesiva obesidad troncal puede subestimar la medición de la DMO.

Se recomienda fuertemente señalar en el informe que la validez de la mediciones en las regiones alteradas por el artefacto se puede ver afectada (Consenso, grado U). 


\section{Informe de densitometría}

1. ¿Qué debe incluir el reporte densitométrico? Se recomienda fuertemente que todo informe de densitometría identifique marca y modelo de densitómetro debido a que existen diferencias que influyen en la resolución, precisión reproducibilidad y escala de los resultados en densidad e imagen (Consenso, grado U).

Informe de características del paciente ${ }^{6}$. Tabla 1.

Se recomienda adicionar detalle del resultado de FRAX actualizado al momento de realizar la densitometría.

Se recomienda reproducir textualmente el informe del FRAX o imprimir el resultado de la herramienta online: https://www.sheffield.ac.uk/FRAX/ tool.jsp?country $=50$ (Consenso, grado A).

Informe de resultados ${ }^{18}$

a. Imágenes

Se recomienda incluir las imágenes del examen incluyendo las mediciones de las vértebras individuales y en bloques, $y$ de los diferentes segmentos de caderas o antebrazo (Consenso, grado A).

Se recomienda fuertemente especificar las limitaciones del estudio incluyendo posibles elementos de distorsión del paciente que puedan afectar la validez de la medición (Consenso, grado U). b. Valores medidos o resultados

Se recomienda que los valores estén expresados en $\mathrm{g} / \mathrm{cm}^{2}$ y $T$ o $Z$-score por sitio de adquisición (Recomendación, GRADE 1) ${ }^{4,6}$ (Tabla 2).

\section{c. Diagnóstico}

Se sugiere incluir un diagnóstico único para el paciente, basado en la DO más baja, en cualquier sitio, y evitar diagnósticos independientes por sitio de medición (Consenso, grado B).

Se recomienda utilizar solamente los siguientes términos: Normal, Osteopenia, Densidad mineral ósea disminuida para la edad, Osteoporosis y Osteoporosis "severa" cuando existe antecedente de fractura por fragilidad (Recomendación, GRADE $1)^{5,6}$.

Se recomienda, si se dispone de un examen previo en el mismo equipo, informar los cambios en porcentaje (Consenso, grado A).

2. ¿Qué no debe incluir el reporte de la DO?

Sugerimos que el reporte de DO no incluya el diagnóstico de osteoporosis u osteopenia en un sitio determinado si no es la conclusión global del estudio (Consenso, grado $C$ ).

Sugerimos que el reporte de densitometría ósea no incluya los términos osteopenia u osteoporosis "leve" o "moderada" (Consenso, grado B).

Recomendamos que en el reporte de densitome-

Tabla 1. Informe de características del paciente en densitometría ósea

\begin{tabular}{|lcc|}
\hline Características & Consenso, grado & Recomendación \\
$\begin{array}{l}\text { Edad } \\
\text { Género }\end{array}$ & $U$ & Se recomienda fuertemente \\
Peso/talla actual & & \\
$\begin{array}{l}\text { Status gonadal/edad menopausia } \\
\text { Diagnóstico clínico } \\
\text { Uso de terapia para osteoporosis }\end{array}$ & $\mathrm{A}$ & Se recomienda \\
\hline
\end{tabular}

Tabla 2. Informe de sitios de medición en densitometría ósea

$\begin{array}{lcc}\text { Sitio } & \text { Consenso, grado } & \text { Recomendación } \\ \text { Columna lumbar debe incluir L1-L4 } & U & \text { Se recomienda fuertemente } \\ \text { Si usa otro segmento, especificar los excluidos y el motivo de ello } & & \text { Se recomienda } \\ \text { Incluir cuello femoral y/o cadera total de ambos lados } & \text { A } \\ \text { En antebrazo considerar el radio 33\% de extremidad no dominante } \\ \text { Especificar detalle del segmento cuando se informan otras áreas }\end{array}$


tría ósea no se utilice el adjetivo "severo", si no hay antecedente de una fractura por fragilidad (Consenso, grado A).

Recomendamos que en el reporte de densitometría ósea no se utilicen los datos del triángulo de Ward o trocánter como criterio de diagnóstico (Consenso, grado A).

Sugerimos que el reporte de densitometría ósea no incluya un plazo recomendado para un próximo examen ni describa que ha habido pérdida ósea sin tener densitometría previa (Consenso, grado $B$ ).

Recomendamos que el reporte de densitometría ósea no incluya recomendaciones a favor o en contra de un tratamiento determinado (Consenso, grado A).

\section{Referencias}

1. Camacho PM, Petak SM, Binkley N, Clarke BL, Harris ST, Hurley DL, et al. American Association of Clinical Endocrinologists and American College of Endocrinology Clinical Practice Guidelines for the Diagnosis and Treatment of Postmenopausal Osteoporosis. Endocr Pract 2016; 22 (4): 1-42.

2. Nelson HD, Haney E, Chou R, Dana T, Fu R, Bougatsos C. Screening for Osteoporosis: Systematic Review to Uptodate the 2002 U.S. Preventive Services Task Force Recommendation. US. Oregon Health and Science University. 2010; p. 1-217.

3. Dasher L, Newton C, Lenchick L. Dual X-ray Absorptiometry in Today`s Clinical Practice. Radiol Clin North Am 2010; 48: 541-60.

4. Lorente Ramos RM, Azpeitia Armán J, Arévalo Galeano N, Muñoz Hernández A, García Gómez JM, Gredilla Molinero J. Absorciometría con rayos X de doble energía. Fundamentos, metodología y aplicaciones clínicas. Radiología 2012; 54 (5): 410-23.

5. International Atomic Energy Agency. Dual Energy X-Ray Absorptiometry for Bone Mineral Density and Body Composition Assessment. Austria. Human Health Series. 2010. p. 1-115.

6. Shepherd J, Schousboe J, Broy S, Engelke K, Leslie W. Executive Summary of the 2015 ISCD Position Development Conference on Advanced Measures From DXA and QCT: Fracture Prediction Beyond BMD. J Clin Densitom 2015;18 (3): 274-86.

7. Shepstone L, Lenaghan E, Cooper C, Clarke S, Fong-Soe-Khioe R, Fordham R, et al. Screening in the community to reduce fractures in older women
(SCOOP): a randomised controlled trial. Lancet 2018; 24: 741-7.

8. Clark P, Denova-Gutiérrez E, Zerbini C, Sánchez A, Messina O, Jaller JJ, et al. FRAX-based intervention and assessment thresholds in seven Latin American countries. Osteoporos Int 2018; 29 (3): 707-15.

9. Curry SJ, Krist AH, Owens DK, Barry MJ, Caughey $\mathrm{AB}$, Davidson KW, et al; US Preventive Services Task Force. Screening for Osteoporosis to Prevent Fractures: US Preventive Services Task Force Recommendation Statement. JAMA 2018; 319 (24): 2521-31.

10. Siris ES, Adler R, Bilezikian J, Bolognese M, DawsonHughes B, Favus MJ, et al. The clinical diagnosis of osteoporosis: a position statement from the National Bone Health Alliance Working Group. Osteoporos Int 2014; 25 (5): 1439-43.

11. Wu CH, McCloskey EV, Lee JK, Itabashi A, Prince R, $\mathrm{Yu}$ W, et al. Consensus of Official Position of IOF/ISCD FRAX Initiatives in Asia-Pacific Region. J Clin Densitom 2014; 17: 150-5.

12. Briot K, Geusens P, Em Bultink, Lems WF, Roux C. Inflammatory diseases and bone fragility. Osteoporos Int 2017; 28 (12): 3301-14.

13. American Gastroenterological Association (AGA) Committee on Osteoporosis in Gastrointestinal Disease. American Gastroenterological Association medical position statement: guidelines on osteoporosis in gastrointestinal diseases. Gastroenterology 2003; 124 (3): 791-4.

14. Romme EA, Geusens P, Lems WF, Rutten EP, Smeenk FW, van den Bergh JP, et al. Fracture prevention in COPD patients; a clinical 5-step approach. Respir Res 2015; 16: 32.

15. Brown TT, Hoy J, Borderi M, Guaraldi G, Renjifo B, Vescini F, et al. Recommendations for evaluation and management of bone disease in HIV. Clin Infect Dis 2015; 60 (8): 1242-51.

16. Panday K, Gona A, Humphrey MB. Medication-induced osteoporosis: screening and treatment strategies. Ther Adv Musculoskelet Dis 2014; 6 (5): 185-202.

17. Cosman F, de Beur SJ, LeBoff MS, Lewiecki EM, Tanner B, Randall S, et al. Clinician's Guide to Prevention and Treatment of Osteoporosis. Osteoporos Int 2014; 25 (10): 2359-81.

18. Dimai HP. Use of dual-energy X-ray absorptiometry (DXA) for diagnosis and fracture risk assessment; WHO-criteria, T- and Z-score, and reference databases. Bone 2017; 104: 39-43.

19. Lera L, Albala C, Ángel B, Sánchez H, Picrin Y, Hormazábal MJ, et al. Anthropometric model for the prediction of appendicular skeletal muscle mass in Chilean older adults. Nutr Hosp 2014; 29 (3): 611-7. 
20. Morgan SL, Prater GL. Quality in dual-energy X-ray absorptiometry scans. Bone 2017; 104: 13-28.

21. McCloskey EV, Oden A, Harvey NC, Leslie WD, Hans D, Johansson H, et al. A Meta-Analysis of Trabecular Bone Score in Fracture Risk Prediction and
Its Relationship to FRAX. J Bone Miner Res 2016; 31 : 940-8.

22. Zeytinoglu M, Jain RK, Vokes TJ. Vertebral fracture assessment: Enhancing the diagnosis, prevention, and treatment of osteoporosis. Bone 2017; 104: 54-65. 